CARNETS DE Carnets de géographes

GÉOGRAPHES.

12 | 2019

Géographie(s) des mobilisations

\title{
De la planète finie aux espaces de vie
}

La dimension spatiale des militantismes pour la décroissance en France et au Québec

Anne-Laure Pailloux

(2) OpenEdition

Journals

Electronic version

URL: http://journals.openedition.org/cdg/4595

DOI: $10.4000 /$ cdg.4595

ISSN: 2107-7266

Publisher

UMR 245 - CESSMA

Electronic reference

Anne-Laure Pailloux, « De la planète finie aux espaces de vie », Carnets de géographes [Online], 12 |

2019, Online since 09 December 2019, connection on 24 September 2020. URL : http://

journals.openedition.org/cdg/4595; DOI : https://doi.org/10.4000/cdg.4595

This text was automatically generated on 24 September 2020.

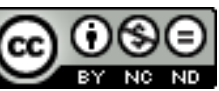

La revue Carnets de géographes est mise à disposition selon les termes de la Licence Creative Commons Attribution - Pas d'Utilisation Commerciale - Pas de Modification 4.0 International. 


\title{
De la planète finie aux espaces de vie
}

\author{
La dimension spatiale des militantismes pour la décroissance en France \\ et au Québec
}

Anne-Laure Pailloux

1 Cette thèse présente l'ambition de comprendre comment la décroissance est pensée, défendue, revendiquée et surtout vécue et mise en pratiques par des militants et militantes français'es et québécois·es. Dans une logique multiscalaire, de la planète finie aux espaces de vie, c'est-à-dire des manières de penser le monde aux manières d'y vivre au quotidien, en passant par les multiples manières de militer pour la décroissance, la thèse propose une analyse de la dimension spatiale des militantismes pour la décroissance dans tous leurs aspects, que ce soit les mobilisations collectives, les modes de structuration et de fonctionnement des organisations, les idées et revendications de la décroissance ou les implications quotidiennes de l'engagement militant.

2 Défendant l'intérêt de prendre au sérieux la cause pour laquelle des individus se mobilisent pour mieux comprendre, en retour, les pratiques militantes, la thèse présente une analyse des idées de la décroissance, de leur appropriation et de leur circulation depuis le début des années 2000 jusqu'à leur constitution en mouvement de pensée politique. Cette recherche positionne ainsi la décroissance au regard de plusieurs courants critiques contemporains tels la justice environnementale, l'écosocialisme, les communs et les pensées critiques de la technique, du travail ou du centralisme démocratique.

Outre cette analyse de corpus théorique, la thèse s'est appuyée sur une enquête ethnographique multi-située de longue durée mêlant observations et entretiens. Cela s'est traduit par l'immersion dans un réseau d'interconnaissance constitué par les militantes et militants engagées dans une organisation politique dite nationale: le Mouvement des Objecteurs de Croissance (MOC) ou le Parti Pour la Décroissance (PPLD) en France et le Mouvement Québécois pour une Décroissance Conviviale (MQDC) au Québec. Au-delà de ces structures, j'ai cherché à observer le plus possible de réunions et de mobilisations auxquelles participaient ces militantes et mis l'accent sur l'analyse 
de leur participation à la mise en place d'un jardin collectif en permaculture dans l'Est francilien.

4 L'étude du répertoire d'action collective des militantes pour la décroissance a mis en lumière son évolution depuis le champ politique vers des actions locales associatives intégrées à l'économie sociale et solidaire. Elle montre le maintien d'actions collectives inscrites dans des registres d'action organisés à l'échelle nationale, notamment l'éducation populaire et la manifestation, mais également la valorisation de registres d'action locale, en particulier en participant aux luttes contre les « grands projets inutiles et imposés » et en initiant des « alternatives concrètes ». L'analyse de la dimension spatiale des actions contestataires révèle le rôle de la mobilité individuelle, des ressources locales et de l'appropriation de l'espace pour faire exister un événement même (inter)national. La focale portée sur le conflit dit de Notre-Dame-des-Landes a dévoilé l'intérêt d'une analyse de la construction scalaire d'une mobilisation étant donnée l'imbrication des échelles en jeu, depuis l'occupation locale jusqu'à la constitution d'un réseau européen de résistance. Elle témoigne également d'une dynamique militante plus large qui articule action locale et enjeux planétaires, en accordant une grande importance à l'expérimentation d'autres manières de vivre au quotidien, comme dans l'espace occupé rebaptisé « zone à défendre ».

5 Ainsi, à l'inverse de registres d'actions mettant en jeu une extension scalaire pour atteindre l'échelle nationale, voire supranationale, c'est-à-dire l'échelle des pouvoirs politiques ou économiques visés, et souvent contestés, les décroissants et décroissantes cherchent à revaloriser l'action locale. En s'engageant dans leur espace de vie, les militantes cherchent à changer immédiatement les conditions matérielles d'existence au quotidien et se trouvent ainsi soumis à des incidences biographiques sur leur mode de vie (par exemple en termes d'habitat, de mobilité ou de (dé)consommation) voire sur leur activité professionnelle. L'analyse des trajectoires d'engagement a en effet permis de faire ressortir le sentiment de cohérence, d'efficacité ou d'utilité sociale accordé à ces actions collectives locales pouvant se traduire, pour plusieurs militantes et malgré le déclassement social que cela impliquait, par des reconversions professionnelles au sein de leurs structures d'engagement.

6 L'étude des effets de l'engagement complétait, dans une approche longitudinale, celle des dispositions à l'engagement politique, des capitaux individuels mobilisés dans les actions collectives et de la socialisation organisationnelle jouée par le collectif. L'analyse $d u$ fonctionnement des organisations politiques et associatives étudiées mettait quant à elle en lumière les rapports de domination qui les structuraient. Dans cette démarche articulant sociologie et géographie sociale critique, je proposais une réflexion sur ma posture de recherche, depuis ma propre position dans ces rapports intersectionnels en jeu pendant l'enquête de terrain jusqu'à la restitution des résultats. Ce souci de réflexivité s'est donné à voir par l'appropriation de l'approche dite des savoirs situés qui postule l'importance de se situer soi-même autant que l'on cherche à situer les « enquêtées » c'est-à-dire à dévoiler ses propres dispositions et objectifs mis en jeu dans la recherche.

7 En conclusion, cette thèse analyse des revendications et des pratiques militantes portées par un petit nombre d'individus dans une démarche de cohérence radicale mais qui sont aujourd'hui appropriées et mises en œuvre par une diversité de collectifs contestataires et alternatifs. Cette recherche éclaire ainsi des préoccupations et pratiques sociales qui la dépassent. 


\section{Discipline}

Géographie

\section{Directeurs}

Jérôme MONNET, Professeur d'aménagement et urbanisme, UPEM

Fabrice RIPOLL, Maître de conférences en géographie, UPEC

\section{Université}

Université Paris-est

Composition du jury de thèse, soutenue le 12 décembre 2016 :

Benoît LEROUX, Maître de conférences en sociologie, Université de Poitiers

(examinateur)

Jérôme MONNET, Professeur d'aménagement et urbanisme, UPEM (directeur de thèse)

Sylvie OLLITRAULT, Directrice de recherche en science politique, CNRS, Rennes (rapporteure)

Fabrice RIPOLL, Maître de conférences en géographie, UPEC (co-directeur de thèse)

Vincent VESCHAMBRE, Professeur de sciences sociales, ENSAL (président du jury)

\section{Situation professionnelle au moment de la publication}

Chercheuse Post-Doctorante à l'Université de Bretagne Sud, EA

\section{Courriel}

alpailloux[@]gmail.com

\section{AUTHOR}

\section{ANNE-LAURE PAILLOUX}

Docteure en géographie, Département Géoarchitecture, Université de Bretagne Sud 